\title{
[18F]Fluorodeoxyglucose accumulation as a biological marker of hypoxic status but not glucose transport ability in gastric cancer
}

Ryusuke Takebayashi ${ }^{1 \dagger}$, Kunihiko Izuishi ${ }^{1,4^{*} \dagger}$, Yuka Yamamoto ${ }^{2}$, Reiko Kameyama ${ }^{2}$, Hirohito Mori ${ }^{3}$, Tsutomu Masaki ${ }^{3}$ and Yasuyuki Suzuki ${ }^{1}$

\begin{abstract}
Background: The use of [18F] 2-fluoro-2-deoxy-D-glucose positron emission tomography (FDG-PET) for detection of gastric cancer is often debated because FDG uptake varies for each patient. The purpose of this study was to clarify the molecular mechanisms involved in FDG uptake.

Material and methods: Fifty patients with gastric cancer who underwent FDG-PET and gastrectomy were studied. Snap-frozen tumor specimens were collected and examined by real-time PCR for relationships between maximum standardized uptake value (SUV) and mRNA expression of the following genes: glucose transporter 1 (GLUT1), hexokinase 2 (HK2), hypoxia-inducible factor 1a (HIF1a), and proliferating cell nuclear antigen (PCNA).

Results: Tumor size was the only clinicopathological parameter that significantly correlated with SUV. Transcripts for the genes evaluated were about three-fold higher in malignant specimens than in normal mucosa, although only HIF1a was significantly correlated with SUV. When divided into intestinal and non-intestinal tumors, there was a significant correlation between SUV and tumor size in intestinal tumors. Interestingly, the weak association between SUV and HIF1a expression in intestinal tumors was substantially stronger in non-intestinal tumors. No correlation was found between SUV and mRNA expression of other genes in intestinal or non-intestinal tumors.
\end{abstract}

Conclusion: SUV was correlated with HIF1a, but not PCNA, HK2, or GLUT1 expression. FDG accumulation could therefore represent tissue hypoxia rather than glucose transport activity for aggressive cancer growth.

Keywords: 18-Fluorodeoxyglucose, Positron emission tomography, Gastric cancer, Glucose transporter-1, Hypoxia-inducible factor $1 a$

\section{Background}

Radiology examinations provide important information for cancer treatment, and [18F] 2-fluoro-2-deoxy-D-glucose positron emission tomography (FDG-PET) differs from conventional imaging through its use of cellular metabolic characteristics to detect a variety of tumors and metastases [1,2]. FDG-PET detection rates tended to vary widely for gastric cancer, however, with $0-44 \%$

\footnotetext{
* Correspondence: izuishi@kms.ac.jp

${ }^{\dagger}$ Equal contributors

'Department of Gastroenterological Surgery, Kagawa University 1750-1, Ikenobe, Miki, Kita, Kagawa 761-0793, Japan

${ }^{4}$ Department of Gastroenterological Surgery, Federation of Public Services and Affiliated Personnel Aid Associations, Takamatsu Hospital, 4-18

Tenjinmae, Takamatsu, Kagawa 760-0018, Japan

Full list of author information is available at the end of the article
}

detection in early stages and $34-94 \%$ detection in advanced stages $[1,3-5]$. Pseudolesions from physiological FDG uptake prevent a more precise diagnosis [6]. Moreover, signet ring cell carcinoma was reported to significantly lower the standardized uptake value (SUV) of FDG compared to papillary or tubular adenocarcinomas $[1,7,8]$. The usefulness of FDG-PET detection for gastric cancer is thus a matter of debate.

Besides detecting tumors based on absolute value, FDG-PET can also assess the response to chemotherapy based on relative values before and after cancer treatment [1]. Previous studies have suggested a significant association between the metabolic changes observed by FDG-PET and clinical or histopathological response [9-11]. One report in particular predicted patient 
prognoses by detecting early changes in glucose uptake after chemotherapy, which could help prevent the continuation of ineffective treatments. Ott et al. found that a reduction in FDG uptake of more than 35\% for metabolic responders predicted a favorable response in gastric cancer patients two weeks after initiation of chemotherapy [11], while metabolic non-responders or FDG non-avid tumors received an unfavorable prognosis.

Cancer cells theoretically require a greater amount of glucose consumption than healthy tissue because of increased cell division $[12,13]$ or anaerobic respiration in tumors [14]. Many cancers increase glucose transport through glucose transporter 1 (GLUT1) and glucose phosphorylation by hexokinase (HK) [15-17]. A correlation between FDG uptake and GLUT1 expression has been found in gastric cancer patients $[1,3,7,8]$, but these studies were conducted by non-quantitative immunohistochemistry analysis, such as negative or positive staining that can vary by evaluator. We therefore evaluated the expression of glucose metabolism-related proteins through quantitative reverse-transcription polymerase chain reaction (qRT-PCR) and compared the results to maximum SUV of FDG-PET. In addition, we also analyzed the expression of proliferating cell nuclear antigen (PCNA) as a valid marker of proliferation [18] and hypoxia-inducible factor 1 alpha (HIF $1 \alpha)$ as a marker of hypoxia [19] to elucidate either of these mechanisms, i.e., tumor proliferation or tumor hypoxia, contribute to FDG uptake. We then discuss the significance and difficulties involved with the clinical application of FDG-PET in gastric cancer due to FDG uptake mechanisms.

\section{Materials and methods Patients}

This retrospective study involved 50 patients (29 male and 21 female; mean age \pm standard error of measurement [SEM], $65.8 \pm 1.4$ years) with gastric cancer who underwent same FDG-PET system before gastrectomy in Kagawa University from July 2005 to March 2010. Tumor specimens were snap-frozen at the time of surgery, and stored at $-80^{\circ} \mathrm{C}$. Participants were divided into 25 cases of intestinal tumors and 25 cases of non-intestinal tumors based on histopathological diagnoses. When focal FDG uptake was not found in the stomach, SUV was calculated from a lesion determined by histology results after gastrectomy. The International Union Against Cancer staging system was used to determine clinicopathological parameters associated with FDG uptake. The protocol was approved by the institutional review board of our institution, and all patients provided written informed consent.

\section{FDG-PET imaging}

FDG-PET images were acquired with a PET scanner (ECAT EXACT HR+, Siemens/CTI, Knoxville, TN, USA).
Patients fasted at least five hours before FDG injection. Images were reviewed on a Sun Microsystems workstation (Siemens/CTI) along transverse, coronal, and sagittal planes with maximum intensity projection images. The images were then interpreted independently by two experienced nuclear medicine physicians blinded to the clinical data. Tumor lesions were identified as areas of focally increased FDG uptake exceeding that of surrounding normal tissue. A region of interest was placed over each lesion to include the highest levels of radioactivity. Maximum SUV was calculated with the following formula: SUV = $\mathrm{cdc} /(\mathrm{di} / \mathrm{w})$, wherein $\mathrm{cdc}$ is the decay-corrected tracer tissue concentration $(\mathrm{Bq} / \mathrm{g})$, di is the injected dose $(\mathrm{Bq})$, and $\mathrm{w}$ is the patient's body weight ( $\mathrm{g}$ ).

\section{Immunohistochemical staining}

Immunohistochemical staining was performed to determine GLUT1 and HK2 levels in gastric cancer tumors. Briefly, resected specimens were fixed in $10 \%$ buffered formalin solution, embedded in paraffin, and sectioned at a thickness of $4 \mu \mathrm{m}$. Slides were then incubated overnight at room temperature with primary rabbit polyclonal antibody against GLUT1 (1:200) or HK2 (1:100). Avidin-biotinperoxidase complex staining was performed according to the manufacturer's instructions (Santa Cruz Biotechnology, CA, USA). Finally, nuclei were counterstained with hematoxylin [20].

\section{Real-time PCR}

Total RNA was isolated from specimens by guanidinium isothiocyanate-acid phenol extraction and quantified by absorbance at $260 \mathrm{~nm}$. Total RNA $(1 \mu \mathrm{g})$ was used for reverse transcription, and the resulting CDNA was analyzed by real-time PCR with Power SYBR Green PCR Master Mix and ABI Prism 7000 (Applied Biosystems, Foster, CA, USA). Target-specific oligonucleotide primers and probes were previously described [20,21]. $18 \mathrm{~S}$ rRNA was used as an endogenous control. Primers and probes for $18 \mathrm{~S}$ rRNA were obtained in a Pre-Developed TaqMan Assay Reagent kit (Applied Biosystems, Stockholm, Sweden).

\section{Statistical analysis}

Data are expressed as mean \pm SEM. Paired SUV results were compared by student's $t$-test. Multiple one-way analysis of variance was used to assess differences in mRNA levels. Correlation analyses were performed with Spearman's correlation analysis test. $\mathrm{P}<0.05$ was considered statistically significant.

\section{Results}

Relationship between mean SUV and clinicopathological data in gastric cancer

Of the 50 gastric cancer lesions, 45 showed focally increased FDG uptake. The majority of patients had 
advanced gastric cancer and a mean tumor size of $7.5 \pm$ $0.5 \mathrm{~cm}$, with 16 cases classified as stage 4 . The mean SUV of stage 4 patients was $9.0 \pm 1.3$, while mean SUV of stage 2 and stage 3 patients combined was $8.3 \pm 0.6$ (Figure 1a). When tumors were divided into intestinal and non-intestinal tumors, mean SUVs were $7.8 \pm 0.7$ and $9.2 \pm 1.0$, respectively (Figure $1 \mathrm{~b}$ ). When divided by median lymph node metastasis, 22 cases had less than three and 28 cases had three or more; mean SUVs were not significant at $9.4 \pm 1.0$ and $7.8 \pm 0.7$, respectively. When divided by maximum median tumor diameter, 22 cases were less than $7.0 \mathrm{~cm}$ and 28 cases were $7.0 \mathrm{~cm}$ or greater; mean SUVs were $7.0 \pm 0.6$ and $9.7 \pm 0.9$, respectively $(\mathrm{P}<0.05)$.

These results indicate that SUV was not dependent on the number of lymph node metastases or cancer stage. Maximum tumor diameter was the only parameter with a significant difference. To more precisely determine its correlation with SUV, we carried out quantitative analysis (Figure 1c). Spearman's correlation analysis indicated a possible relationship between the factors ( $\mathrm{rs}=0.33$, $\mathrm{P}<0.05)$.

\section{Expression of glucose transporter and glucose metabolizing enzymes in gastric cancer}

GLUT1 staining was seen in the cell walls, while HK2 staining was observed in the cytoplasm, of tubular (Figure 2a1, 2b1) and poorly differentiated (Figure 2a2, 2b2) adenocarcinomas. Based on these results, specimens were evaluated by qRT-PCR to determine the expression of glucose metabolism-related genes (HK1, HK2, GLUT1, and glucose-6-phosphatase (G6Pase)). HK2 and GLUT1 levels were three-fold higher in cancerous tissue than in normal mucosa $(\mathrm{P}<0.001)$ (Figure 2c). G6Pase is a gluconeogenic enzyme in the liver that reverses the reaction metabolized by HK (glucose to glucose-6-phosphate) [22]. Its expression appeared to decrease in cancerous tissue, but not to a significant degree. In spite of the high levels, no significant correlation was observed between SUV and HK2 (Figure 2d) or GLUT1 (Figure 2e) expression. The glucose metabolic pathway in cancerous tissues may be too complicated to regulate with the alteration of a single molecule.

\section{Relationship between mean SUV and HIF1a or PCNA expression in gastric cancer}

To determine whether tumor proliferation or tumor hypoxia contributes to FDG uptake, PCNA expression was analyzed as a proliferation marker and HIF1 $\alpha$ expression as a hypoxia marker. The mRNA levels for both genes were about three-fold higher in cancerous cells than in normal mucosa $(\mathrm{P}<0.001)$ (Figure $3 \mathrm{a})$. To more precisely determine the association of SUV with PCNA and HIF1 $\alpha$ mRNA expression, their correlation was quantitatively analyzed. There was no correlation between PCNA expression and SUV (Figure 3b), but HIF1 $\alpha$ expression was correlated to SUV by Spearman's correlation analysis ( $\mathrm{rs}=0.53, \mathrm{P}<0.01$ ) (Figure 3c). There was no correlation between PCNA expression and HIF1 $\alpha$ expression (data not shown).
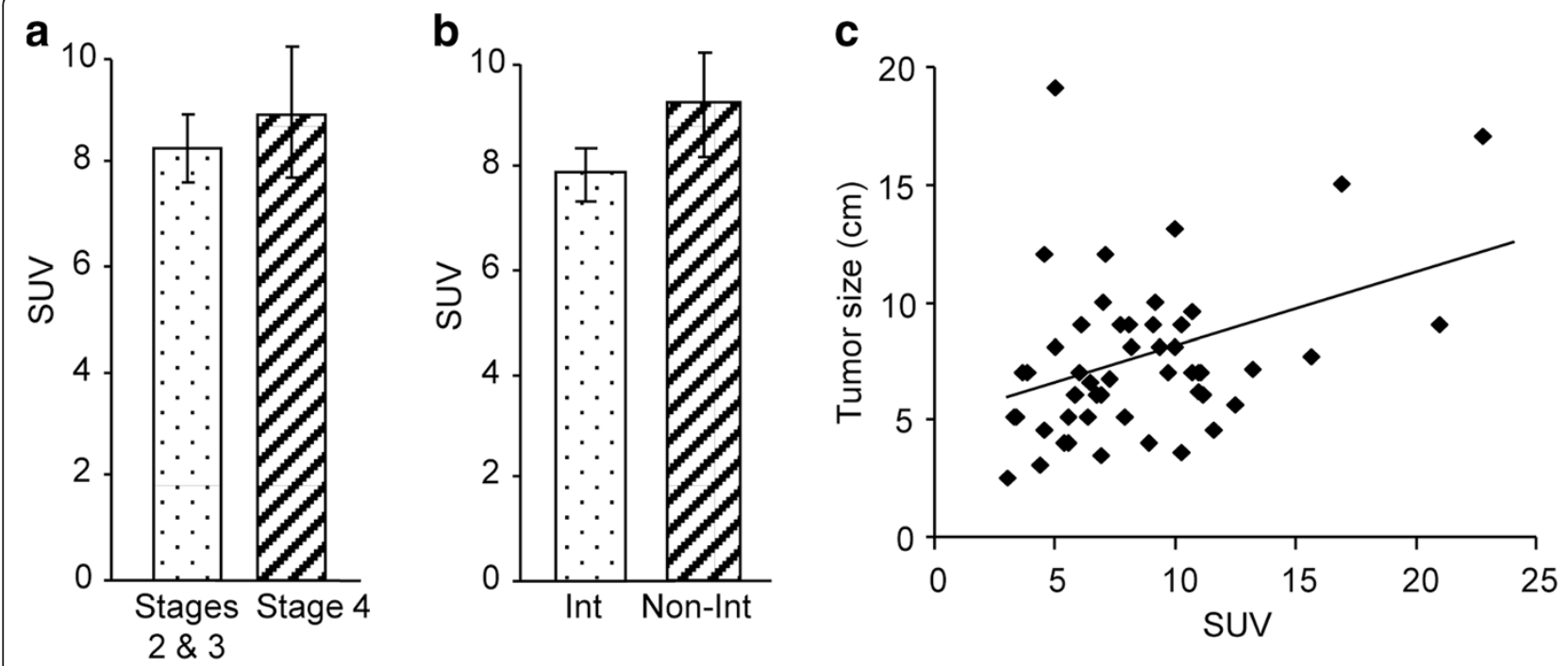

Figure 1 Relationship between mean standardized uptake value and clinicopathological data in gastric cancer. (a) Mean standardized uptake value (SUV) in stage 4 gastric cancer patients was not significantly higher than in stage 2 and stage 3 patients. (b) Mean SUV in intestinal tumors was not significantly greater than in non-intestinal tumors. (c) Spearman's correlation analysis revealed a significant correlation between tumor size and mean SUV ( $r$ s $=0.33, \mathrm{P}<0.05)$. Values are expressed as mean \pm SEM. Int; Intestinal Type, Non-Int; Non-intestinal Type, SUV; Standardized Uptake Value. 

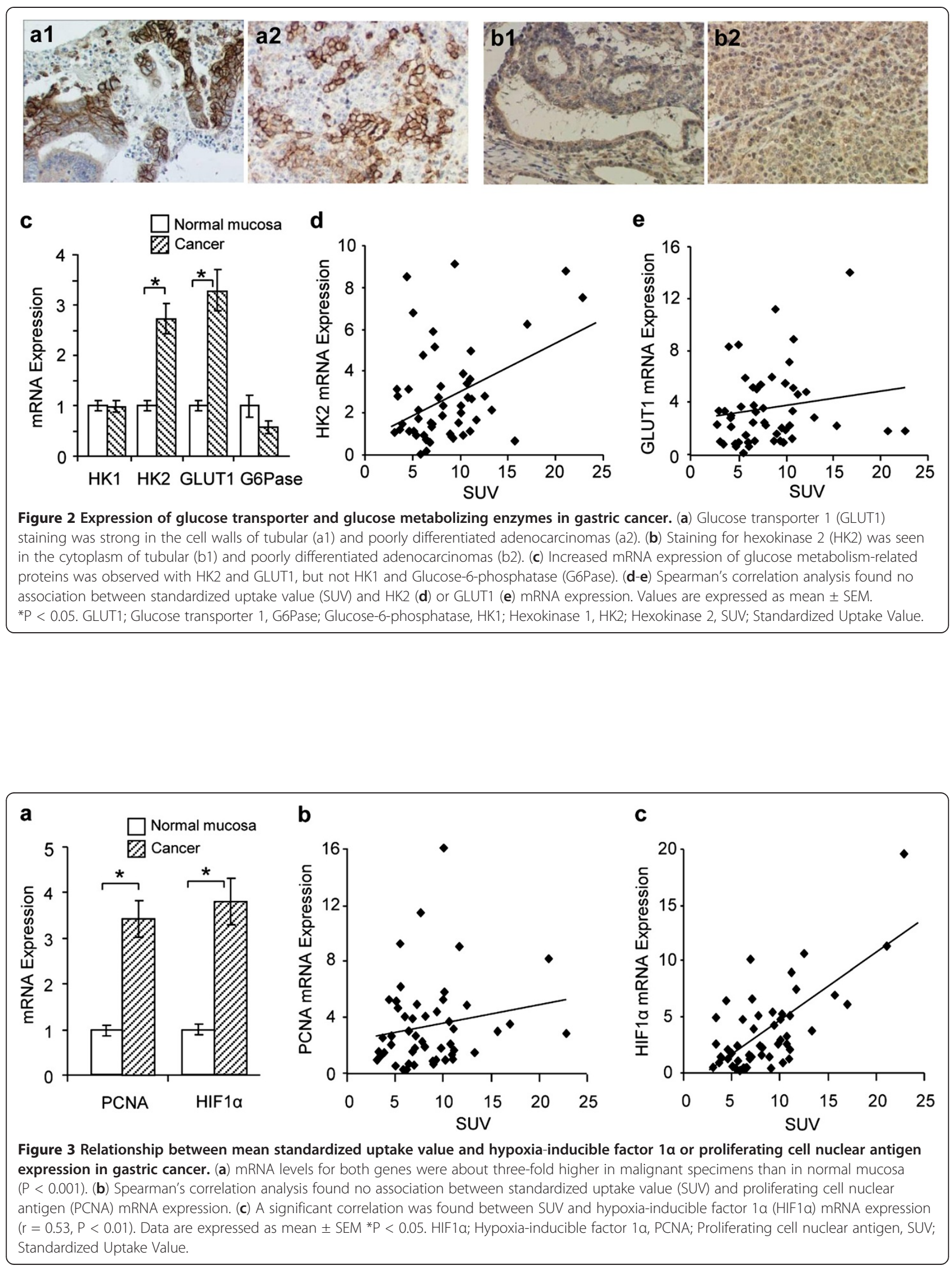
Expression of HK1, HK2, GLUT1, and G6Pase mRNA levels in intestinal and non-intestinal gastric cancers

Although HK1 mRNA levels were similar, HK2 mRNA levels were higher in both specimen types compared to normal mucosa $(\mathrm{P}<0.01)$. GLUT1 expression was significantly higher in intestinal specimens than in normal mucosa $(\mathrm{P}<0.01)$, but was unchanged in non-intestinal specimens (Figure 4). PCNA and HIF1 $\alpha$ expression increased three-fold in intestinal tumors $(\mathrm{P}<0.01)$ compared to normal mucosa.

\section{Correlation between mean SUV and tumor size, HIF1a mRNA levels, or PCNA mRNA levels in intestinal and non- intestinal gastric cancers}

To examine factors associated with SUV in intestinal and non-intestinal gastric cancers, their correlation was quantitatively analyzed. Spearman's correlation analysis indicated a possible relationship between SUV and tumor size in intestinal specimens $(\mathrm{rs}=0.50, \mathrm{P}<0.05)$ (Figure 5a), but not non-intestinal specimens (Figure $5 \mathrm{~d}$ ). The correlation between HK2 or GLUT1 expression and SUV did not find in both cancers (data not shown). There was no correlation between SUV and PCNA mRNA expression in either cancer type (Figure $5 \mathrm{~b}$ and $5 \mathrm{e}$ ). Interestingly, the weak association between SUV and HIF1 $\alpha$ mRNA expression in intestinal specimens ( $\mathrm{rs}=0.48$, $\mathrm{P}<0.05$ ) (Figure 5c) was stronger in non-intestinal specimens $(\mathrm{rs}=0.56, \mathrm{P}<0.01)$ (Figure $5 \mathrm{f}$ ).

\section{Discussion}

FDG-PET has been used to not only detect cancerous lesions, but also predict therapeutic response after chemotherapy $[1,11,23]$. There are several possible mechanisms behind its ability to reveal malignant potential or cancer cell activity. Our results found that SUV in stage 4 gastric cancer patients was no higher than in stage 2 and stage 3 patients, and the main tumor SUV did not reflect the number of lymph node metastases. Only tumor size was associated with SUV, a correlation also reported in breast, pancreatic, and colorectal cancers [20,24,25]. These finding narrow the FDG-PET mechanism possibilities by suggesting that SUV reflects tumor size rather than tumor cell activity for each cancer stage.

\section{Over expression of glucose metabolism-related protein in tumors}

A molecular explanation for high FDG uptake in cancerous tissues is the overexpression of GLUT1, the molecule reported to be responsible for FDG uptake in various cancers $[20,26]$. Glucose uptake ability as assessed by FDG-PET was significantly correlated with the doubling time of tumors [27] because increased uptake can provide additional energy to support tumor growth. Yamada et al. [7] determined from immunohistochemistry that GLUT1 expression was an important factor for FDG uptake and also a prognostic tool for gastric cancer. Alakus et al. [3] similar reported that FDG uptake in gastric cancer is dependent on the degree of GLUT1 staining. Our immunohistochemical staining also showed strong GLUT1 expression in cell membranes, as well as GLUT1 mRNA expression 3.3-fold greater in tumors than the surrounding mucosa; however, Spearman's correlation analysis did not find a relationship between GLUT1 expression and SUV. HK2 also plays an important role in FDG catabolism, with its overexpression significantly associated with

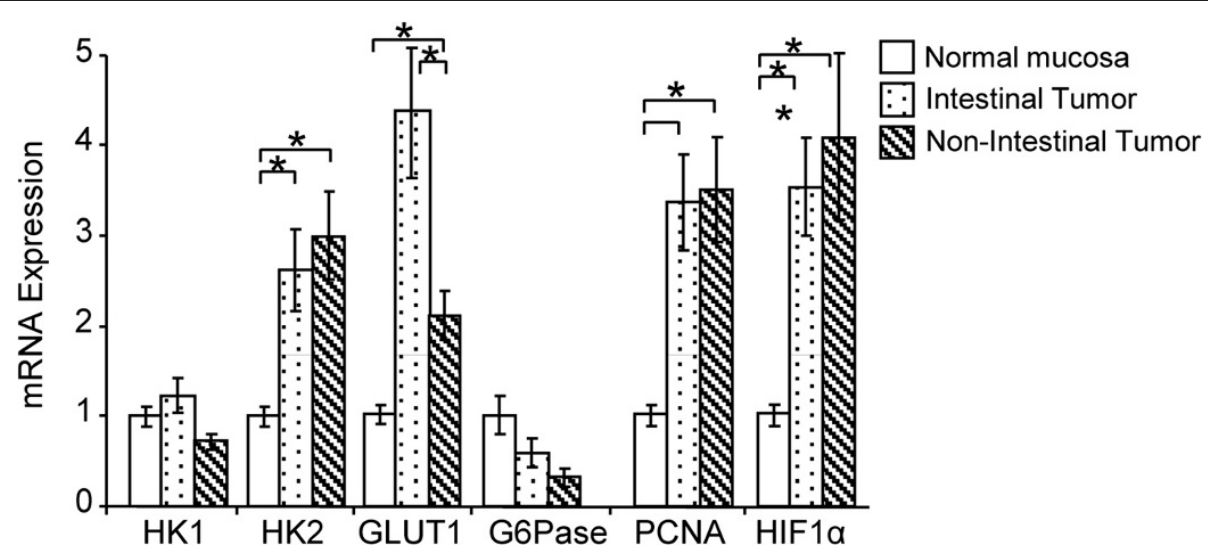

Figure 4 Expression of glucose metabolism-related proteins in intestinal and non-intestinal gastric cancers. Hexokinase 1 (HK1) mRNA levels were similar to those in normal mucosa, while HK2 mRNA levels were higher in both intestinal and non-intestinal gastric cancers $(P<0.01)$. Glucose transporter 1 (GLUT1) expression increased more in intestinal tumors than in normal mucosa $(P<0.01)$, but were unchanged in nonintestinal tumors. Glucose-6-phosphatase (G6Pase) expression decreased, but the difference was not significant. The mRNA expression of proliferating cell nuclear antigen (PCNA) and hypoxia-inducible factor 1a (HIF1a) increased more than three-fold compared to normal mucosa $(P<0.01$ ). Data are expressed as mean \pm SEM *P < 0.05 (ANOVA). GLUT1; Glucose transporter 1, G6Pase; Glucose-6-phosphatase, HIF1a; Hypoxiainducible factor 1a, HK1; Hexokinase 1, HK2; Hexokinase 2, PCNA; Proliferating cell nuclear antigen, SUV; Standardized Uptake Value. 


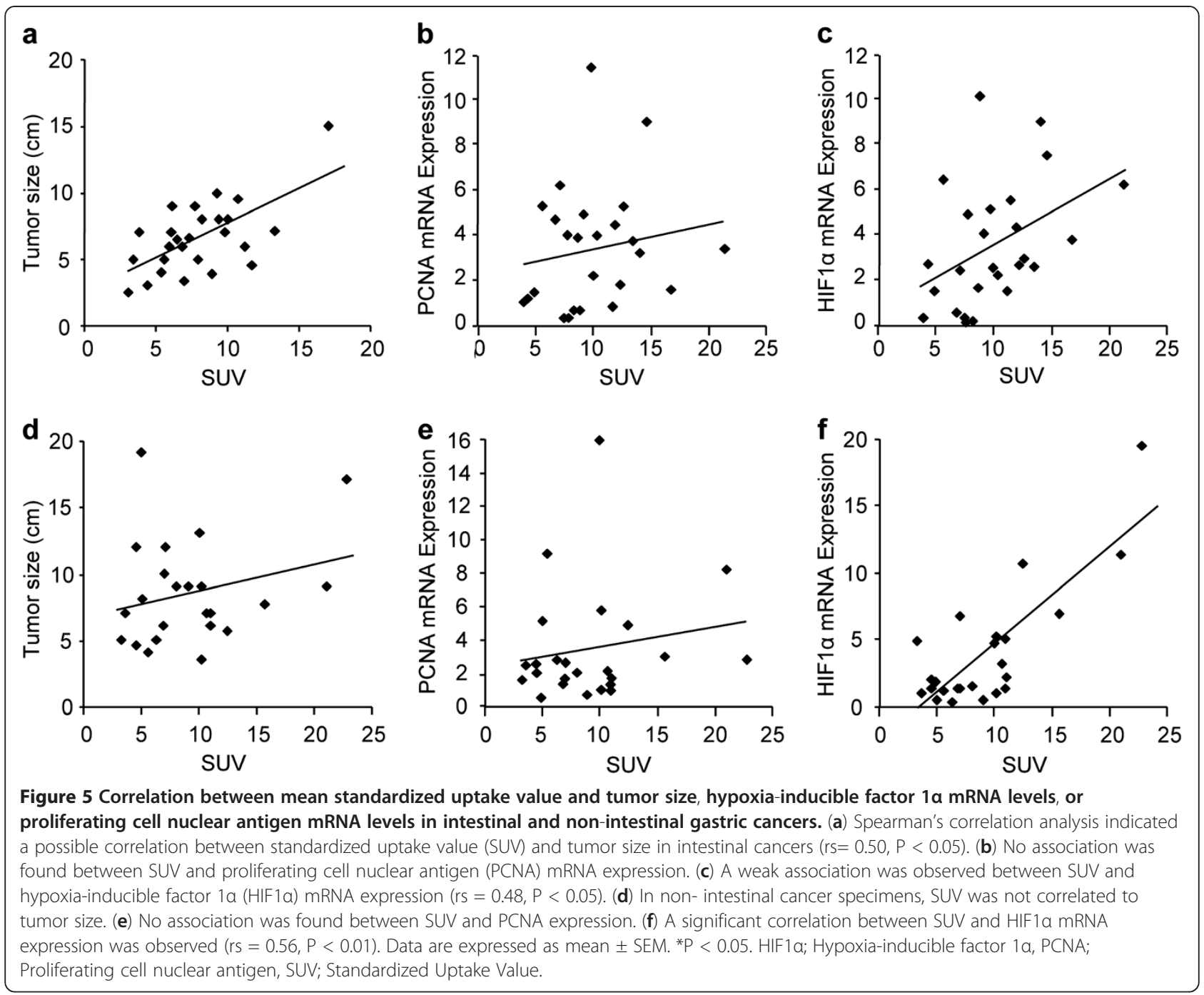

SUV in malignant tumors [15,28]. We also found HK2 overexpression in gastric cancer tumors, but there was again no correlation between HK2 expression and SUV. Other complicated mechanisms, such as blood flow, accumulation of inflammatory cells, and cellularity might be also contribute to the intensity of FDG uptake based on malignant energy demand [20].

\section{Hypotheses of the increased glucose uptake in tumor}

Two major hypotheses have been presented to explain the increased glucose uptake in cancerous tissue, either that enhanced glucose consumption is associated with tumor proliferative activity $[12,13]$ or that tissue hypoxia induces anaerobic glycolysis to increase glucose metabolism [14]. Our results indicate that FDG uptake associated significantly with hypoxia, reflected by HIF1 $\alpha$ expression, but not with proliferative activity, reflected by PCNA expression; these gastric cancer findings correspond to our previous report on colorectal cancer [20]. Rapid cancer growth induces a hypoxic environment in tumors. HIF1 $\alpha$ acts as a sensor for hypoxic stress and upregulates angiogenic factors and promotes transcription of several genes, including glucose transporters and glycolytic enzymes such as GLUT1 and HK, for tumor survival [29]. HIF1 $\alpha$ may also be involved with oncogenic alterations to glucose metabolism because it activates cancer-related gene transcription and affects pathways such as angiogenesis, cell survival, glucose metabolism, and cell invasion [30]. HIF1 $\alpha$ overexpression has been associated with increased patient mortality rates in several cancers, while inhibited expression reduced tumor growth in an in vitro study [30]. HIF1 $\alpha$ could thus play a central role in cancer progression that FDG uptake represents.

\section{Histological differences in the expression of glucose metabolism-related proteins}

The non-intestinal gastric cancers, signet ring cell carcinoma and mucinous carcinoma, presented a very low 
FDG uptake compared to their intestinal counterparts due to low GLUT1 expression [1,3,7,8]. Berger et al. reported that FDG-PET revealed an unusually high percentage $(41 \%)$ of false-negative results in carcinoma with mucin. There was a positive correlation of FDG uptake with tumor cellularity but a negative correlation with the amount of mucin [31]. Therefore, non-intestinal gastric cancers, which have characters of low cellularity and/or high mucin content, do not show high FDG uptake. Alakus et al. has reported that over expression of GLUT1 in papillary/tubular adenocarcinoma and signet ring cell carcinoma was $94 \%$ and $24 \%$, respectively [3]. Our results also indicate that GLUT1 expression in nonintestinal cancers was lower than in intestinal cancers. However, the reason why such aggressive cancers showed low GLUT1 expression is unknown. A previous study found that glutamine metabolism is upregulated in gastric cancer [32]. Gastric cancer cells use glutamine as an energy source in a hypoxic tumor microenvironment, which may eliminate the necessity for glucose transport. This metabolic alteration accompanied with malignant transformation has been reported in other cancers [33]. Interestingly, a glutamine-based PET is being developed; if successful, this contradiction could be disproved in the future.

On the other hand, HIF1 $\alpha$ expression correlated with SUV in both types, although a more significant correlation was seen in non-intestinal specimens. The nonintestinal tumors may have been influenced more by hypoxia derived from tumor fibrosis due to a scattering tumor growth pattern than hypoxia due to increased tumor size. Further research will be needed to determine the exact reason.

\section{Limitations of this study}

There are several limitations in our study. First, we examined 50 cases of gastric cancer patients. The fewness of cases affects the statistical analysis and makes it difficult to get firm results in association of FDG uptake and the expression of the proteins. Second, we could not exclude the possibility of contribution of physiological FDG uptake in normal stomach on cancerous lesion. Finally, our results did not show the direct physiological relationship between HIF1 $\alpha$ as a marker of hypoxic condition and FDG accumulation.

\section{Conclusions}

The usefulness of FDG-PET in the detection of malignant tumors or prediction of prognoses has been widely reported. However, our results indicate that the degree of FDG accumulation does not always suggest a prognosis in gastric cancer. This study is the first to show the correlation by evaluating FDG uptake in a quantitative manner. Upregulation of glucose transport due to increased GLUT1 expression was not an explanation for the different FDG uptakes observed, although tumor hypoxia and HIF1 $\alpha$ expression may provide a reasonable mechanism. Further investigation is needed to confirm these results, but metabolic alternation through HIF1 $\alpha$ induction in tumor hypoxia could increase FDG uptake in gastric cancer.

\section{Competing interests}

The authors declare that they have no competing interests.

\section{Authors' contributions}

RT: Analyzing data, experimental work, and drafting article. Kl: Conception, design, experimental work, and acquiring data. YY: Acquiring and analyzing data of FDG-PET. RK: Acquiring and analyzing data of FDG-PET. HM:

Acquiring clinical data. TM: Revising the manuscript, and statistical analysis. YS: Enhancing its intellectual content. All authors read and approved the final manuscript.

\section{Acknowledgements}

We are extremely grateful to all the clinical staff who cared for these patients. We also are thankful to Dr. Shoji Kimura for his reliable experimental suggestion.

\section{Author details}

'Department of Gastroenterological Surgery, Kagawa University 1750-1, Ikenobe, Miki, Kita, Kagawa 761-0793, Japan. ²Department of Radiology, Kagawa University 1750-1, Ikenobe, Miki, Kita, Kagawa 761-0793, Japan. ${ }^{3}$ Department of Internal Medicine of Gastroenterology and Neurology, Faculty of Medicine, Kagawa University 1750-1, Ikenobe, Miki, Kita, Kagawa 761-0793, Japan. ${ }^{4}$ Department of Gastroenterological Surgery, Federation of Public Services and Affiliated Personnel Aid Associations, Takamatsu Hospital, 4-18 Tenjinmae, Takamatsu, Kagawa 760-0018, Japan.

Received: 14 February 2013 Accepted: 22 May 2013

Published: 29 May 2013

\section{References}

1. Shimada H, Okazumi S, Koyama M, Murakami K: Japanese gastric cancer association task force for research promotion: clinical utility of ${ }^{18} \mathrm{~F}$-fluoro-2-deoxyglucose positron emission tomography in gastric cancer. A systematic review of the literature. Gastric Cancer 2011, 14:13-21.

2. Murakami K: FDG-PET for Hepatobiliary and pancreatic cancer: advances and current limitations. World J Clin Oncol 2011, 2:229-236.

3. Alakus H, Batur M, Schmidt M, Drebber U, Baldus SE, Vallböhmer D, Prenzel KL, Metzger R, Bollschweiler E, Hölscher AH, Mönig SP: Variable 18Ffluorodeoxyglucose uptake in gastric cancer is associated with different levels of GLUT-1 expression. Nucl Med Commun 2010, 31:532-538.

4. Chen J, Cheong JH, Yun MJ, Kim J, Lim JS, Hyung WJ, Noh SH: Improvement in preoperative staging of gastric adenocarcinoma with positron emission tomography. Cancer 2005, 103:2383-2390.

5. Mochiki E, Kuwano H, Katoh H, Asao T, Oriuchi N, Endo K: Evaluation of 18F-2-deoxy-2-fluoro-D-glucose positron emission tomography for gastric cancer. World I Surg 2004, 28:247-253.

6. Kamimura K, Nagamachi S, Wakamatsu H, Fujita S, Nishii R, Umemura Y, Ogita M, Komada N, Sakurai T, Inoue T, Fujimoto T, Nakajo M: Role of gastric distention with additional water in differentiating locally advanced gastric carcinomas from physiological uptake in the stomach on 18F-fluoro-2-deoxy-D-glucose PET. Nucl Med Commun 2009, 30:431-439.

7. Yamada A, Oguchi K, Fukushima M, Imai Y, Kadoya M: Evaluation of 2-deoxy-2-[18F]fluoro-D-glucose positron emission tomography in gastric carcinoma: relation to histological subtypes, depth of tumor invasion, and glucose transporter-1 expression. Ann Nucl Med 2006, 20:597-604

8. Kawamura T, Kusakabe T, Sugino T, Watanabe K, Fukuda T, Nashimoto A, Honma K, Suzuki T: Expression of glucose transporter-1 in human gastric 
carcinoma: association with tumor aggressiveness, metastasis, and patient survival. Cancer 2001, 92:634-641.

9. Ott $K$, Herrmann $K$, Krause BJ, Lordick F: The value of PET imaging in patients with localized gastroesophageal cancer. Gastrointest Cancer Res 2008, 2:287-294

10. Ott K, Herrmann K, Lordick F, Wieder H, Weber WA, Becker K, Buck AK, Dobritz M, Fink U, Ulm K, Schuster T, Schwaiger M, Siewert JR, Krause BJ: Early metabolic response evaluation by fluorine-18 fluorodeoxyglucose positron emission tomography allows in vivo testing of chemosensitivity in gastric cancer: long-term results of a prospective study. Clin Cancer Res 2008, 14:2012-2018

11. Ott K, Fink U, Becker K, Stahl A, Dittler HJ, Busch R, Stein H, Lordick F, Link T, Schwaiger M, Siewert JR, Weber WA: Prediction of response to preoperative chemotherapy in gastric carcinoma by metabolic imaging: results of a prospective trial. J Clin Oncol 2003, 21:4604-4610.

12. Adler LP, Blair HF, Makley JT, Williams RP, Joyce MJ, Leisure G, al-Kaisi N, Miraldi F: Noninvasive grading of musculoskeletal tumors using PET. J NuCl Med 1991, 32:1508-1512.

13. Avril N: GLUT1 expression in tissue and (18)F-FDG uptake. J Nucl Med 2004, 45:930-932.

14. Vander Heiden MG, Cantley LC, Thompson CB: Understanding the Warburg effect: the metabolic requirements of cell proliferation. Science 2009, 324:1029-1033.

15. Paudyal B, Oriuchi N, Paudyal P, Tsushima Y, Higuchi T, Miyakubo M, Ishikita T, Nakajima T, Endo K: Clinicopathological presentation of varying $18 \mathrm{~F}-$ FDG uptake and expression of glucose transporter 1 and hexokinase II in cases of hepatocellular carcinoma and cholangiocellular carcinoma. Ann Nucl Med 2008, 22:83-86.

16. Khan MA, Combs CS, Brunt EM, Lowe VJ, Wolverson MK, Solomon H, Collins BT, Di Bisceglie AM: Positron emission tomography scanning in the evaluation of hepatocellular carcinoma. J Hepatol 2000, 32:792-797.

17. Miyakubo M, Oriuchi N, Tsushima Y, Higuchi T, Koyama K, Arai K, Paudyal B, lida Y, Hanaoka H, Ishikita T, Nakasone Y, Negishi A, Mogi K, Endo K: Diagnosis of maxillofacial tumor with L-3-[18F]-fluoro-alphamethyltyrosine (FMT) PET: a comparative study with FDG-PET. Ann Nucl Med 2007, 21:129-135.

18. Baserga R: Growth regulation of the PCNA gene. J Cell Sci 1991, 98:433-436

19. Hong SS, Lee H, Kim KW: HIF-1alpha: a valid therapeutic target for tumor therapy. Cancer Res Treat 2004, 36:343-353.

20. Izuishi K, Yamamoto Y, Sano T, Takebayashi R, Nishiyama Y, Mori H, Masaki T, Morishita A, Suzuki Y: Molecular mechanism underlying the detection of colorectal cancer by 18F-2-fluoro-2-deoxy-D: -glucose positron emission tomography. J Gastrointest Surg 2012, 16:394-400.

21. Kameyama R, Yamamoto Y, Izuishi K, Sano T, Nishiyama Y: Correlation of 18F-FLT uptake with equilibrative nucleoside transporter-1 and thymidine kinase- 1 expressions in gastrointestinal cancer. Nucl Med Commun 2011, 32:460-465.

22. Kuang Y, Schomisch SJ, Chandramouli V, Lee Z: Hexokinase and glucose- 6 -phosphatase activity in woodchuck model of hepatitis virus-induced hepatocellular carcinoma. Comp Biochem Physiol C Toxicol Pharmacol. 2006, 143:225-231.

23. Di Fabio F, Pinto C, Rojas Llimpe FL, Fanti S, Castellucci P, Longobardi C, Mutri $V$, Funaioli C, Sperandi F, Giaquinta S, Martoni AA: The predictive value of 18F-FDG-PET early evaluation in patients with metastatic gastric adenocarcinoma treated with chemotherapy plus cetuximab. Gastric Cancer 2007, 10:221-227

24. Heudel P, Cimarelli S, Montella A, Bouteille C, Mognetti T: Value of PET-FDG in primary breast cancer based on histopathological and immunohistochemical prognostic factors. Int J Clin Oncol 2010, 15:588-593.

25. Izuishi K, Yamamoto Y, Sano T, Takebayashi R, Masaki T, Suzuki Y: Impact of 18-fluorodeoxyglucose positron emission tomography on the management of pancreatic cancer. J Gastrointest Surg 2010, 14:1151-1158.

26. Usuda K, Sagawa M, Aikawa H, Ueno M, Tanaka M, Machida Y, Zhao XT, Ueda $Y$, Higashi K, Sakuma T: Correlation between glucose transporter-1 expression and 18F-fluoro-2-deoxyglucose uptake on positron emission tomography in lung cancer. Gen Thorac Cardiovasc Surg 2010, 58:405-410.

27. Duhaylongsod FG, Lowe VJ, Patz EF Jr, Vaughn AL, Coleman RE, Wolfe WG: Lung tumor growth correlates with glucose metabolism measured by fluoride-18 fluorodeoxyglucose positron emission tomography. Ann Thorac Surg 1995, 60:1348-1352.

28. Ong LC, Jin Y, Song IC, Yu S, Zhang K, Chow PK: 2-[18F]-2-deoxy-D-glucose (FDG) uptake in human tumor cells is related to the expression of GLUT1 and hexokinase II. Acta Radiol 2008, 49:1145-1153.

29. Dang CV, Semenza GL: Oncogenic alterations of metabolism. Trends Biochem Sci 1999, 24:68-72.

30. Semenza GL: Targeting HIF-1 for cancer therapy. Nat Rev Cancer 2003, 3:721-732.

31. Berger KL, Nicholson SA, Dehdashti F, Siegel BA: FDG PET evaluation of mucinous neoplasms: correlation of FDG uptake with histopathologic features. AJR Am J Roentgenol 2000, 174:1005-1008.

32. Hirayama A, Kami K, Sugimoto M, Sugawara M, Toki N, Onozuka H, Kinoshita T, Saito N, Ochiai A, Tomita M, Esumi H, Soga T: Quantitative metabolome profiling of colon and stomach cancer microenvironment by capillary electrophoresis time-of-flight mass spectrometry. Cancer Res 2009, 69:4918-4925.

33. Rajagopalan KN, DeBerardinis RJ: Role of glutamine in cancer: therapeutic and imaging implications. J Nucl Med 2011, 52:1005-1008.

doi:10.1186/1756-9966-32-34

Cite this article as: Takebayashi et al:: [18F]Fluorodeoxyglucose accumulation as a biological marker of hypoxic status but not glucose transport ability in gastric cancer. Journal of Experimental \& Clinical Cancer Research 2013 32:34.

\section{Submit your next manuscript to BioMed Central and take full advantage of:}

- Convenient online submission

- Thorough peer review

- No space constraints or color figure charges

- Immediate publication on acceptance

- Inclusion in PubMed, CAS, Scopus and Google Scholar

- Research which is freely available for redistribution 